\title{
High-Reliability Organizing (HRO) and Abrupt Change from COVID 19: Failure of Scientific Rationality and Classical Logic
}

Daved van Stralen, MD, FAAP, Thomas A. Mercer, RAdm, USN

\begin{abstract}
Neonatologists built the science of neonatology with logic and rationality. The cognitive processes of scientific rationality and classical logic, developed for a structured environment, are poorly defined for operational use. The first impairments from even mild uncontrollable stress are in the cognitive and memory areas of the brain. A 'cosmology' event collapses our sensemaking even as we believe in our own rationality. The novice does not have personal anecdotes that are protective in such a collapse. When applying the abstractions of logic to a particular concrete situation, we can fulfill our own sensemaking, or we can make sense for the good of the community. It is the premise and logic that differ. The dominant narrative from scientific rationality and classical logic has become privileged with the loss of knowledge gained during risky operations. Such lost knowledge creates inaccurate models with longlasting effects on education and policy. In the operational environment, lost knowledge can kill.
\end{abstract}

\section{"Neonatologists built the science of neonatology from scientific rationality and classical logic. It was gaining life through the extension of neonatology to smaller and sicker babies developed with different forms of rationality and other logic systems."}

\section{Introduction}

Neonatologists built the science of neonatology from scientific rationality and classical logic. It was gaining life through the extension of neonatology to smaller and sicker babies developed with different forms of rationality and other logic systems. This is more than a gap between theory and practice (1); it is a gap between the normative stance of a spectator at a fixed point outside the flux of events and the pragmatic stance of a participant engaging the turbulence from within the trajectory of events (2). The liminal threshold beyond which the pragmatic stance operates conceals the rationality and system of logic used by operators. The operator neuromodulates to reduce stress and fear and draws on methods for reasoning with imperfect information in flux. Otherwise, events cascade toward failure.

The obstetrician hands a premature newborn baby to the neonatal team. With the heart rate dropping, the nurse vigorously dries and stimulates the baby, the heart rate increases. The respiratory care practitioner (RCP) gives supplemental oxygen, thrusts the jaw forward, and starts bag-mask ventilation; the heart rate again decreases. Tension is more than emotional; it is physical as the baby is pulled by each caregiver. The physician intubates the infant. The heart rate becomes normal and steady. The baby is now active.

\section{"Tension is more than emotional;" it is physical as the baby is pulled by each caregiver. The physician intubates the infant. The heart rate becomes normal and steady. The baby is now active."}

The NICU charge nurse enters the delivery room, and the RCP immediately explains how the nurse missed the need for intubation. The nurse follows with an explanation that the baby needed drying and stimulation-the RCP counters with the need for intubation, evidenced by the pediatrician intubating the baby.

The pediatrician (the author, DvS) stated, "I intubated because the heart rate dropped too low, but the baby had responded to drying and stimulation." The charge nurse countered, "The nurse is new. We have to get to know you before we can trust you."

"I came from the fire department. We had a saying; If you don't trust me, then you can't be trusted. We trusted new people because we knew we could carry them when they needed it. We had the strength and trust in ourselves that we could carry our partner if needed. That's how you go into danger." The pediatrician was startled to note everyone in the room staring at him. The labor nurses approached and said thank you.

Note in this vignette the sources and reliability of the information, the use of reasoning and cognition, and the influence of stress and fear. Each participant believed in themselves, was only a few feet apart, yet inferred different conclusions, made different decisions, then acted in opposition. Each believed in their own rationality and logical conclusions. Yet each was different.

A high-risk situation, or any exigency, necessitates interaction to generate information while also creating structure. With information, we transform abstract concepts into concrete actions. We infer useful conclusions from vague signals or developing circumstances. Reasoned evaluation supports how we think to decide, decide to act, and modify those actions. Then stress and threat mix this up.

Education, planning, rules, and protocols for exigencies such as this all go into the cognitive and memory parts of the brain. The first impairments from even mild uncontrollable stress are in the

NEONATOLOGY TODAY is interested in publishing manuscripts from Neonatologists, Fellows, NNPs and those involved in caring for neonates on case studies, research results, hospital news, meeting announcements, and other pertinent topics.

Please submit your manuscript to: LomaLindaPublishingCompany@gmail.com 
brain's same cognitive and memory areas. While the participants in the vignette identified the problem of bradycardia, interpretations differed between hypothermia from evaporative water loss versus bradycardia from hypoxemia. Linking these abstract concepts to the concrete situation failed. An element of fear abruptly drives efforts to keep distance from the threat observed in the form of blaming and accusation or by deflection and justification (3). To keep distance from the threat (rapid bradycardia) for selfpreservation, the participants explained their own actions and the actions of the others. Information was lost.

\section{"Rationality and reason, along with} rules and protocols, derive support from classical logic. We are accustomed to linearity, deduction and analysis, the law of the excluded middle, and the law of noncontradiction."

Rationality and reason, along with rules and protocols, derive support from classical logic. We are accustomed to linearity, deduction and analysis, the law of the excluded middle, and the law of non-contradiction. In the vignette above, time branched with a course of different possible events between drying with stimulation and hand ventilation with intubation. Beliefs were revised as new information was incorporated or the circumstances changed. A binary truth value did not exist as each circumstance and action could be interpreted right or wrong depending on where they occurred. We began evaluation, but the series of contingencies from each treatment on the other treatment confounded interpretation. Rationality and reason from classical logic were lost.

What appears strongly "right" during the time before an abrupt disruption, based on cognitive science and classical logic, becomes irrelevant amid the exigency. This article describes how the environment of as experienced creates practical rationality and the logic of practice. The mental reaction to the environment creates a gap between scientific rationality and classical logic. This gap does not become visible through investigation or interrogation.

\section{Logic as the use of information}

We need relevant information to ensure that our actions match our observed reality and known concepts, reduce error, and confirm the consistency of these concepts. We need relevant information when we purposely risk error to investigate inconsistencies and contradictions between observed reality and known concepts. We strengthen our knowledge with deduction and the analysis of new information. We create knowledge through induction for synthesis when we encounter new information. Information reassures us that we are on a safe path or prepares us at the edge of a crisis. Information reveals contradictions for further investigation. Neonatologists have used the information to extend their experience to help smaller and sicker neonates (4). How we use information has a great influence on how we achieve HRO.

As a medical discipline, neonatology makes use of information through scientific rationality, a system of analytic methodology, classical logical inference, and detached observation ((5), 93-95); (6). Amartya Sen, economics Nobel laureate, described rationality as subjecting one's decisions to reasoned scrutiny rather than following formula (7), 3-5. Using classical logic, which relies on true premises, we preserve truth, not falling into the acceptance of falsity. Scientific rationality creates a representational world that we can transport to any circumstance. However, the abstraction of a representational world is neither the actual world nor necessarily a possible world. It is only a representation of the actual world.

In the field of uncertainty and threat, we must reliably use information. We must avoid the trap of a false belief that our subconscious wants to be true. We must avoid the seduction of logical inference that appears to prove our belief. The abstraction of a representational world can be applied anywhere. We must not, however, sacrifice our real-world for the tractability of discrete concepts and classical logic.

\section{"In the field of uncertainty and threat, we must reliably use information. We must avoid the trap of a false belief that our subconscious wants to be true. We must avoid the seduction of logical inference that appears to prove our belief."}

\section{Uncertainty and logic}

This becomes a fundamental problem for implementing and using $\mathrm{HRO}$ - in high risk, how do we rationally use the information and generate reasoned inferences from that information when our information is imperfect, unstable, unavailable, and the time to act is limited. Academicians offer theory within the normative stance, yet the NICU is a place of practice from the pragmatic stance. Through engagement, we can bridge the gap between theory and practice (1), discrete concepts and continuous perceptions (8), and the normative stance and pragmatic stance (2). The academic lexicon, though, may not provide effective contributions for that engagement.

Note the concepts and wording in the academic and professional literature for decision making: cognition, intuition, rational, rationality, logic, analysis, heuristics, biases, error, complexity, chaos, and information processing. Then recall the NICU environment: uncertainty, time compression, stress, emotions, and doubt. Ambiguity may lead us to construct a world that is not true while supported by evidence (9). In the realm of uncertainty and ambiguity, an error has critical functions for decision-making and safety. Error corrects heuristic bias (10). Error, unfortunately, has become an indicator of careless or poor performance to engender fear of the system and supervisors. The inability to neuromodulate the resulting endemic stress-fear-threat (3) corrupts safe and effective operations, creating the ecology of fear (11).

The structured, academic presentation of theory and logic does not match the experience of Neonatologists, particularly in relation to their years of experience. Perhaps we do not sense the futility or the effects of stress on our cognition, rationality, and logic because of the shifting baseline of experience and what we perceive as normal (12). We draw upon anecdotes from early in our careers to evaluate changes and provide context. Mark those infants who will now live and think of all the lives gained. Anecdotes from our experience provide the mental and emotional stability for reflective reasoning and give meaning to the work of the Neonatologist $(4,13)$. We accept as a baseline the survival and death for infants of certain gestational ages and weights at the beginning of our careers. With experience, we see these babies who previously would have died now thrive. These babies give value to what neonatology has accomplished. The extension of neonatology to gain life is remembered through personal anecdotes triggered by 


\section{"The novice does not have those anecdotes. Should a baby die, the novice experiences a life cut short while the experienced Neonatologist counteracts the pain with knowledge of lives gained that were once lost."}

The novice does not have those anecdotes. Should a baby die, the novice experiences a life cut short while the experienced Neonatologist counteracts the pain with knowledge of lives gained that were once lost. This unrecognized stress impairs the rationality of the novice, engenders a beginning sense of futility, and impairs their ability to infer optimism amid critical illness.

Rather than 'gained life,' the novice comes to expect babies to live who would have died at the beginning of the veteran Neonatologist's career.

During the first years of a new PICU, staffing came from ward nurses and NICU respiratory care practitioners (RCP). Several days before Christmas, in the third year of the PICU, 24 infants received mechanical ventilation due to RSV bronchiolitis. At the end of patient care rounds, the level of agitation of the staff became palpable. Finally, the question was asked, "Why can't we wean these babies and extubate them so they can be home by Christmas?" The question was not about a response to treatment. It emerged from days spent with frightened, anxious parents, limited information and prediction, and the lack of a logical system for understanding. Staff did not have personal anecdotes that would give meaning to their work. The author (DvS) had personal experience with the abrupt death of infants from RSV and had worked to keep these infants alive, un-intubated, pending transfer to a PICU. Thus, the question from the author, "Where did these infants go three years ago? I was at the regional receiving PICU. We did not see infants from this area." Sometimes we must use our own anecdote to shift the baseline. "These 24 infants would have died three years ago, just days before Christmas. They are alive today and will go home to their parents because of you."

We had a similar problem with Adult Respiratory Distress Syndrome (ARDS), a disease new to the PICU. After a few weeks of treating ARDS, staff began to urge PICU attendings to encourage parents to withdraw ventilator support as the ethical response. Again, we sometimes must artificially shift the baseline with an anecdote. The apparent deterioration from tension pneumothorax indicated a healing lung. We counted the children with ARDS, those who lived, and those who died. Our survival rate of $50 \%$ was the national average, but those who lived transferred to the ward after about a month while those who died lingered on the ventilator for 5-7 months. The ones who died were easier to remember because of their longer admission. We were saving the lives of children who otherwise would have died.

The intellectual strength and power of cognitive science, scientific rationality, and classical logic readily give way to the mental effects of novelty, uncertainty, and uncontrollability. Psychological stress is the neuroanatomic reaction, with even minor uncontrollable stress impairing abstract thought, memory retrieval, work- ing memory, cognitive flexibility, and executive functions (14-17). Organizations invest heavily in the objective, structured systems, rely on cognitive flexibility, and presume classical logic will suffice. Yet, even minor stress corrupts their plans while the logic we can use in turmoil is not well identified.

Within the trajectory of turmoil, we must use information. To not use or distrust information is a decision about its use. The search for and evaluation of information delays action even as confirmed information changes or becomes unreliable. Methods of rationality and logic in a stable environment with well-structured problems offer little guidance. People revert to their "common sense." Karl Weick (18) describes how such a 'cosmology' event collapses our sensemaking even as we believe in our own rationality.

Regardless of physical threats and social interactions, we respond to our mental environment in a way that makes sense to us at the moment but becomes refined with reflection as we gain experience. We likely call this "common sense" or "reasoned expertise." Common sense, rationality, reasoning, and logic become subsumed into an individual's logic of practice (6). Scientific rationality guides interpretation and understanding, but we must not forget this is objective and reasoned (7). Formal logical systems for truth become difficult when truth shifts or is contingent. Linear classical logic, separated from experience, influences thinking during the experience.

\section{The Mental Environment of HRO}

Logic and rationality, developed for a structured environment, are poorly defined for operational use. A threshold divides the stable, structured environment from more tumultuous circumstances.

However, what is stable and structured for one person may be tumultuous for the individual a foot away. The threshold dividing these worlds is both physical and mental.

Compared to concrete action, superficial comparison of abstract thought readily puts abstract thought at a higher level of function. This makes sense for abstraction as the depth of reflection versus the immediacy of concreteness. Combining concreteness with action, as Aristotle did, creates a much higher level of thought, the first of Aristotle's Cardinal Virtues (19). When applying abstractions to a particular concrete situation, we can fulfill our own sensemaking, or we can make sense for the good of the community. The former is a vice, the latter a virtue(19). It is the premise and logic that differ.

\section{"Rationality and logic are sufficiently developed for stable states that we become accustomed to relying on them for emergencies, despite the differences in premises, truth values, and the rules of inference. Unfortunately, operators may not necessarily agree on which premises to use, their truth value, and whether an inference is possible."}

Rationality and logic are sufficiently developed for stable states that we become accustomed to relying on them for emergencies, despite the differences in premises, truth values, and the rules of inference. Unfortunately, operators may not necessarily agree on 
which premises to use, their truth value, and whether an inference is possible. At its most basic, we disagree and may disagree quite emotionally. At a deeper and more fundamental level, those with more extensive experience will stop talking.

I had the rear-door position. My weapon was drawn and ready. When the main force went in the front door, I heard yelling and a warning that "they" were running out the back towards another agent and me. When the back door flew open, I was in the ready position (weapon up) and prepped to announce ourselves, etc. That lasted for about $1 / 2$ a second as the first thing out the door was a snarling and angry German Shepherd - which launched right at me and, when I ducked, went right over me and kept on trucking. The suspect was next out, and he was moving almost as fast and was past me before I could react. I yelled for him to stop, but he was a fast-mover. Could I / Should I have shot him in the back - for a drug offense of unknown size/quantity - no way - and I didn't see a weapon.

Richard Holbrook, Special Agent, Air Force Office of Special Investigations (AFOSI), joint narcotics raid with local law enforcement assistance, 1984.

An officer-involved shooting, 11:30 pm, limited moonlight. The victim, a 17-year-old male, lay on the driveway in front of a "garage in the rear." The suspect in a rape assault, he had wielded a knife in a knife-fighter stance running toward a police officer. Examining the victim, I noticed numerous entry wounds in the shins, with a few in the thigh from a shotgun. "I thought you said you shot him," I said to the officer. "I did. But when I saw he was a kid, I aimed for the legs. I didn't want to shoot a kid."

Daved van Stralen, 1973

\section{"The rationality and logic used by both operators a decade apart are not described in the academic literature and not amenable to laboratory study."}

The rationality and logic used by both operators a decade apart are not described in the academic literature and not amenable to laboratory study. More seriously, it is not shared across the liminal threshold with those who have underfed experience in the environment of threat, time compression, existential threat, and the obligation to do the right thing.

We must not rely on a predictable, linear flow or trajectory of events, even during a rapid deterioration. In our experience, "punctuated events" abruptly occur to create seemingly unconnected situations. Karl Wieck (personal communication) observed these "punctuated events" need "punctuated sensemaking," the ability to rapidly create a sense of new events almost independent of the most recent experience.

"During a crisis, there is not time to think about each specific bit of knowledge or experience that we depend on to make sense of imperfect information and ambiguity. But having those resources immediately accessible in our minds, we use them in a conceptual decision-making process to frame the decision. We essentially quickly come up with a paradigm of how to solve the problem. It is after the fact that we retrospectively begin to attribute specific reasons for the decisions that we made."
Capt. Chesley "Sully" Sullenberger (personal communication)

The environment of VUCA-2T

Elsewhere we have described the environment of practice (20), that is, the experienced environment we have in common with military operations, public safety, neonatology, critical care, medical care, nursing care, respiratory care, dietetics, K-12 education, social work, and pediatric subacute care. We have worked closely with all these programs with a robust human behavior component in both internal and external environments, a distinguishing feature from socio-technical systems. We call this environment VUCA-2T.

The military concept of "VUCA" describes the new global environment at the end of the Cold War $(21,22)$ - volatile, uncertain, complex, and ambiguous.

Volatility comes from rapid and abrupt changes in events.

Uncertainty describes the lack of precise knowledge about the situation.

Complexity refers to a large number of interconnected and changing parts coming together.

- $\quad$ Ambiguity describes the multiple interpretations, causes, or outcomes for one situation.

VUCA, however, is a military concept. Military professionals carry the implicit assumption that they work in a dangerous and lethal environment. Consequently, the concept of threat is not translated into civilian applications (23).

Threat impairs cognition and decision-making.

Discussions between a special group in SOCOM (Special Operations Command) and the author (DvS) led to the separation of time compression from volatility. Time compression has meaning and carries special information about the environment, distinguishing these events from urgency or time dependence (23, 24). The group now uses VUCA-2T or VUCA-T ${ }^{2}$ (Sean McKay, personal communication) (25).

Time compression describes the limitations on acquiring information, deciding, or acting before consequential changes in circumstances. Time compression is not a quality of time dependence or time-limitation.

"The liminal zone is the space between the world we know and the world we do not. Old rules do not apply, we have not learned new rules, and we do not know what rules will work."

\section{The liminal environment}

VUCA-2T fits the anthropological experience of liminality as a transition (26) and the operational environment of the HRO. The liminal zone is the space between the world we know and the world we do not. Old rules do not apply, we have not learned new rules, and we do not know what rules will work. This magnifies the gap between theory and practice (1), discrete concepts and continuous perceptions (27), abstractions and concreteness (Karl Weick, personal communication), and the static normative stance and the pragmatic stance from within the trajectory of events (2). 
We can infer information in these situations. For that, we must reduce affective responses to threat in known ways, such as acceptance and control of the feeling of fear (20), neuromodulation of stress-fear-threat (3), and social organizing to prevent the ecology of fear (11). Behaviors come in suites coordinated for various purposes $(28,29)$. These suites of behaviors create sustained, coordinated defensive responses for survival. The primary defensive and survival functions are 1) reflexive, subcortical actions, 2) hindering memory systems from limiting cognition, and 3) volitional behaviors directed toward self-preservation.

Unlike a suite of behaviors, an ensemble of behaviors is learned and is selectively activated by specific threat cures-the ensemble functions for the individual. The behaviors in an ensemble are considered only in relation to the whole, unlike a suite of behaviors where behaviors are considered as a single property with an ecological function. An ensemble of behaviors serves a social and cultural function (30) by maintaining continuity and ongoing social interaction by evoking social support and validating responses from others in ongoing social interaction (31).

\section{"The organized grouping of stress and fear behaviors gives them a semblance of rationality, making them difficult to identify or isolate. Consequently, individuals with maladaptive stress ensembles resist change. Without change, the affective responses as suites of stress behaviors change to behavioral ensembles through operant conditioning"}

The organized grouping of stress and fear behaviors gives them a semblance of rationality, making them difficult to identify or isolate. Consequently, individuals with maladaptive stress ensembles resist change. Without change, the affective responses as suites of stress behaviors change to behavioral ensembles through operant conditioning. Maladaptive behavioral ensembles disrupt rationality to become normalized. Agitation, operant anger, and selfprotection become leadership styles and management tools that create the ecology of fear $(3,11)$.

It becomes difficult to distinguish rational and logical reasoning from stress and fear once they become normative. The inability to translate cognitive and affective processes for VUCA-2T, liminality, and HRO theory into the practice of reliability and safety comes with the loss of nuance and missed, subtle cues within the environment (2). Unrecognized is the loss of neuromodulation as a skill and how to acquire the skill.

\section{Mental responses}

We can adopt rationality as defined by Sen (above) with the caveat that we can have 'reasoned scrutiny,' but the reason that is bounded by time and constrained cognition. What is needed for explication and translation of $\mathrm{HRO}$ are methods to infer meaningful and relevant information from uncertainty and ambiguity.

We paraphrase Vanessa Heggie's (32) comments about the science for climbing Mount Everest: predicting what will happen to a premature infant is a matter of life or death - inaccurate models can kill. Consequently, Neonatologists prioritize neonatal respiratory physiology, not in the laboratory but in the NICU.
The mental responses in this environment, the rationality, and the methods for logical inference are not accessible across the threshold. They remain unstudied and under-described except for the disconnected first-person literature of reflective experience. Often this rationality and logic is reflexively rejected by outsiders and spectators. For operators, the acceptance of uncertainty and fear is unique (20), capability calibrates the threat response (3), and operators focus on possibility over probability. The more significant delineator between spectator and operator is the willingness to say, "I don't know."

"Often this rationality and logic is reflexively rejected by outsiders and spectators. For operators, the acceptance of uncertainty and fear is unique (20), capability calibrates the threat response (3), and operators focus on possibility over probability."

The mental environment as experienced has profound effects on rationality and logic, whether effective or putative. As John Hickey, LAFD engineer, advised the author (DvS), "Davey, it's OK to say, 'I don't know." A powerful and practical basis for inductivism in an environment at the time of major fires and recent deaths of firefighters. In the fire service during that era, "I don't know" yielded trust. Yet, in healthcare, such a statement lowers respect and invites a barrage of disconnected, almost random, advice.

Information in all vignettes presented in this article is incomplete and in flux. The meaning of information is contingent on other information and changes in rapid succession. The capabilities of those accompanying the protagonist have a direct effect on rational reasoning and inference.

Probabilities influence deductive rational inference and decisionmaking. However, during a VUCA-2T event, the individual becomes part of the problem $(2,13)$ with direct influence on probabilities. When treating patients with poor responses to treatment, staff often echoed the question of parents - "What are the chances of survival?" One author (DvS) would stress that the job of the PICU team was to change those probabilities. If the child had a $20 \%$ survival, we work to make it $40 \%$. If $40 \%$ survival, we work to make it $60 \%$. Regardless of the percent survival, we work to put our patients into the survival side, approaching 100\%. This avoids the unspoken situation of some caregivers quietly reducing their effort as the chance of success becomes the chance of failure followed by resignation and the emotion of failure. Shifting toward the creation of a new probability also shifts thinking from passive cognition to more effective motor cognition (4), Weick's (33) "We think by acting."

\section{Thoroughbred racehorses}

The author (DvS) worked with a partner on the fire rescue ambulance who bet at the races. He made as much money at the racetrack as he did from the fire department. He studied each jockey and horse and how they ran against each other in all combinations. The author asked, "But wouldn't the jockey hold back once to change the odds and then make more money?" "No," he answered, "If you hold back once, then the horse does not know when to do it next time. The horse will hold back at the wrong time. A thoroughbred racehorse knows only one way to run - full strength." 
The author shared this story with the staff. The mustang looks wild and free but cannot win the race. The thoroughbred racehorse is disciplined and runs with full strength. In the PICU, we are the thoroughbred.

The author used this metaphor when staff became weary, emotionally depleted, or showed hidden biases or prejudices. Staff did not realize how prejudice and emotional depletion act in negative feedback to create more depletion. Having witnessed complete exhaustion in moments of dire survival, the authors became familiar with the power of that final positive push.

We have only one way to run - full strength.

The dominant account: Planning and rules

Planning to reduce the mental workload during a VUCA-2T event seems prudent.

A police officer led a team of police officers into a building during an active shooter incident. As he moves closer to the shooter, the shots became louder with the stronger smell of gunpowder. Reaching a set of double doors in the hallway, he could not tell if the shots he heard several seconds before came from the cafeteria behind the double doors or around the hallway corner ahead. As he reaches the double doors, another shot rang out, and it was now clear the shot was around the corner ahead and to the left. Moving forward towards the corner with several officers, he noticed several other officers from his team behind him, attempting to open the double doors to confirm what threat was behind them. The leader led rather than directed movement from a fixed point in the hallway. They were not executing the exact communication, movement, cornering, or clearing techniques from training. Coming to a T-intersection in the hallway, he noticed a second team from a different entry arriving around the corner. Natural verbal and visual signaling rather than formal, structured statements avoided the friendly fire. The team around the corner crossed the hallway in front of his team, moving towards the suspect. The suspect had died by suicide.

The recognition of not following "trained techniques" only came to him during my debrief with him. During the event, they simply handled problems.

Steve Papenfuhs, Sergeant, San Jose Police Department (retired)

The authors, including the above author, have interviewed civilians, EMS, firefighters, and law enforcement personnel after a shooting incident. As with this officer, none of the individuals followed the prescribed response (34). They "simply handled problems." However, the responders to a terrorist shooting effectively evacuated 14 shooting victims in 18 minutes without loss of life $(24,35)$, and school staff, following a school shooting, quietly evacuated about 400 elementary school children before the arrival of the first law enforcement officer (36).

We must not confuse being around danger with being in it. Sci-

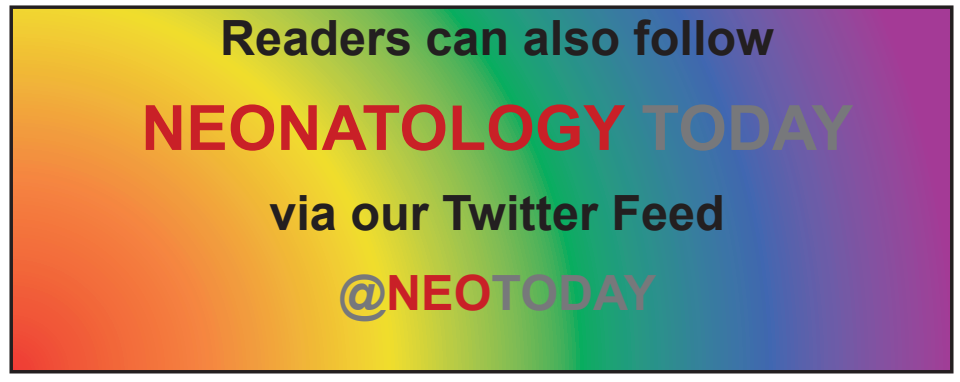

entific rationality and classical logic decontextualize observation and engagement, forming conclusions not tested in the environment. Such conclusions become a substantial portion of the organization's knowledge, constraining actions in confusing situations (37). Nevertheless, the experienced veteran responds to concrete circumstances, but abstract, 'rational' constraints conflict the novice from effective action. However, the narrative of both follows the dominant, 'rational' account $(38,39)$. Positive feedback from these narratives strengthens the dominant account, influencing actions followed by further conforming, though inaccurate, narratives and increasing ignorance of the environment (37).

The dominant narrative from scientific rationality and classical logic becomes privileged. To spectators, the actions of the experienced veteran then appear irrational, lacking discipline $(2,18$, 37-39). Novices follow the dominant 'rational' methods, shifting the baseline from the environment to the office ("laboratory"). The lost knowledge creates inaccurate models with long-lasting effects on education and policy. In the VUCA-2T environment, lost knowledge can kill.

The linearity and sequencing of rules and plans produce reason and logic, distracting from the abstract character of rules and plans created apart from concrete reality. The expert following the rules performs poorly (40-43). We must guard against false protective mechanisms such as abstractions, rules, false confidence, or self-admiration.

\section{Formal Logic Systems}

Proper reasoning permits the identification of valid patterns of inference while at the same time abstracting their content. The field of symbolic logic derives from Aristotle's theory of reasoning. We want to connect ideas and observations through reason in a manner that preserves truth. Formal logic systems confirm ideas and infer new ideas while preserving truth. Validity preserves truth by logical necessity; that is, applying the rules of inference to true premises means the conclusion must follow and be true. Validity reflects the accuracy of form that we cannot have a false statement for our conclusion if the premises are true. If the premises are false, the conclusion is still valid; it is simply not a true conclusion. Validity does not ensure true premises; validity only ensures that the premises will lead to a valid conclusion.

An argument is shown to be valid through derivation. A proof is a formal derivation with stated premises, and each non-premise statement in the proof must be true by a specific rule of inference. Therefore, the conclusion in a proof must be true. In a proof, ev- 
ery line must be true, making the proof valid. On the other hand, if the premises are false, the argument can be valid since validity depends solely on its form; it is just no proof.

The roots of proof are from Late Latin proba, meaning «a test.» proof of the pudding shall be in the eating (1708). Today's version is "The proof is in the pudding" (44).

Formal logic systems should only allow us to derive valid conclusions and verify all valid assertions. They have a similar structure: a set of propositions, apply rules of inference, then derive a conclusion to find a truth statement. An argument is derivable if a conclusion can be reached from its premises. An argument is valid if that conclusion cannot be false, given that the premises are true. Derivability and validity form the basis of soundness, completeness and consistency, critical principles in a formal logic system, and Kurt Gödel's incompleteness theorems.

\section{"Derivability and validity form the basis of soundness, completeness and consistency, critical principles in a formal logic system, and Kurt Gödel's incompleteness theorems."}

Soundness. The system must prove only true sentences. An argument is derivable only if it is valid; only valid formulas can be derived. No deduction can take the argument from true premises to a false conclusion. Thus, deductions preserve truth with the claim that everything derivable in the system is true.

Completeness. The system can prove all valid formulas, the converse of soundness. An argument is valid only if it is derivable. A formal system is complete if, for every statement, either the statement or its negation can be derived (proved) in the system. The deductive system can provide a deduction for every valid argument. The form of completeness differs between logic and math where, in math, it becomes the basis for Gödel's Completeness and Incompleteness Theorems.

Consistency. The system contains no contradictions and contains at least one situation when all formulas are true.

Inconsistency. There is no statement with its own negation that both can be derived within the system. Inconsistency permits a formal system to derive every statement, which means the logic system is meaningless. Triviality in logic describes meaninglessness.

A formal system of logic has completeness, consistency, and effective axiomatization (necessary for decidability).

\section{Kurt Gödel, completeness, and incompleteness (45)}

To have a set of consistent axioms (never a contradiction) and complete (all formulas can be proved) would give mathematics a solid foundation. Gödel used the above concepts to demonstrate that such a foundation is impossible; any foundation for mathematics would be incomplete, there will always be truths that cannot be proved or disproved. He also proved that any set of axioms necessary for decidability could prove its own consistency. Gödel proved there can be no unification of mathematical theory. What can be proved depends on one's starting assumptions.

The Completeness Theorem. Every valid logical expression is provable. That is, every logical expression is either satisfiable or refutable. First-order logic is semantically complete but not syntactically complete.

First incompleteness theorem. Any consistent formal system within which a certain amount of elementary arithmetic can be carried out is incomplete. This only concerns derivability, not provability.

The Second Incompleteness. The consistency of arithmetic cannot be proved by arithmetic.

Truth and falsity are incompatible. Every proposition is either true or false (law of excluded middle); there is no gradation. The same proposition cannot be both true and false at the same time (law of non-contradiction). We can produce a truth table with a two-value logic, but the proposition has neither a value between truth and falsity nor both simultaneously.

The two principles excluded middle and contradiction, along with identity, form the traditional three "laws of thought." Once thought to be independent of each other, Alfred North Whitehead and Bertrand Russell (46) use the principles of logic to deduce the 'law' of (non)contradiction ((46),77, 111 equation 3.24) from the 'law' of the excluded middle ((46), 101 equation 2.11 , (47)). Thus, the two laws are equivalent rather than independent.

To better appreciate how classical logic supports the science and practice of neonatology and resuscitation, we will present a few principles of metalogic, that is, the metatheory of logic. Along with the above elements, a formal logic system demonstrates completeness, consistency, and decidability (axiomatization); the first

Table: A formal system of logic possesses decidability, completeness, and consistency (45)

\begin{tabular}{|l|l|l|}
\hline Decidability & Completeness & Consistency \\
\hline Formalized system & The law of the excluded middle & The law of non-contradiction \\
\hline $\begin{array}{l}\text { Axioms and the proof relation to } \\
\text { derive conclusions }\end{array}$ & $\begin{array}{l}\text { Every property or statement of the } \\
\text { system can be derived from within the } \\
\text { system }\end{array}$ & $\begin{array}{l}\text { No statement and its negation can be derived within } \\
\text { the system }\end{array}$ \\
\hline $\begin{array}{l}\text { All formal and symbolic logic } \\
\text { systems }\end{array}$ & $\begin{array}{l}\text { Incompleteness (50) } \\
\text { - an element cannot be completely } \\
\text { classified } \\
\text { - incomplete or partial information } \\
\text { - the excluded middle is not enforced }\end{array}$ & $\begin{array}{l}\text { Inconsistency (51) } \\
\text { - inconsistent premises will yield any well-formed } \\
\text { statement } \\
\text { - inferential explosion of triviality } \\
\text { - triviality, little importance because any proposition follows from a contradiction } \\
\text { can be inferred }\end{array}$ \\
\hline
\end{tabular}


two are germane to logic that tolerates contradictions and does not enforce the excluded middle $(48,49)$.

\section{Induction and deduction}

Logic supports empirical science. Empirical science develops by evidence from the senses or builds from experience. Logical inference extends evidence in the creation of knowledge while distinguishing truth from falsity. The ability to prove or disprove the properties of knowledge is fundamental in our use of knowledge. This ability lies at the heart of inductive reasoning and deductive reasoning and influences the selection of formalized logic systems.

\section{"Inductivism and inductive reasoning build knowledge from observation, but in inductivism, knowledge is not truth. Conclusions from inductive reasoning are plausible rather than having the certainty of truth we see with deductive reasoning."}

Inductivism and inductive reasoning build knowledge from observation, but in inductivism, knowledge is not truth. Conclusions from inductive reasoning are plausible rather than having the certainty of truth we see with deductive reasoning. The strength of inductive reasoning comes from the relentless pressure to confirm the plausible conclusion, described by Leonhard Euler (52) in George Pólya (53):

"[Observations] will lead us continually to new properties which we shall endeavor to prove afterwards. The kind of knowledge which is supported only by observations and is not yet proved must be carefully distinguished from the truth; it is gained by induction as we usually say.... Indeed, we should use such a discovery as an opportunity to investigate more than exactly the properties discovered and to prove or disprove them; in both cases, we may learn something useful."

Euler believed knowledge could be inductively "assured of its truth" by the number of examples: "anybody can satisfy himself of its truth by as many examples as he may wish to develop. And since I must admit that I am not in a position to give it a rigorous demonstration, I will justify it by a sufficiently large number of examples" $(52,53)$.

The pursuit of inductive confirmation in this fashion can lead to error which then could lead to harm. During the more deliberate extension of a discipline, we can identify and correct inductive errors to reduce the effects of harm (4). Note that in uncertainty, acting or not acting and engaging or not engaging can be equally harmful. In uncertain situations where a consequence includes death, inductive reasoning to identify 'probably correct' conclusions engenders vulnerability, which paradoxically increases safety (20). On the other hand, deductive reasoning seems a better approach as the conclusion is guaranteed to be true. However, this is only if the premises are true. We miss the falsity as the safety margin is only putative. The premises for deductive reasoning must be correct.

It is impossible to deductively treat a premature infant, even with knowledge of anatomy and physiology. The pulmonary system is straightforward because it is included in all science classes and physicians are well-versed in physiology. This can lead to overconfidence by a non-Neonatologist.
Insufficient oxygen in the airways constricts pulmonary arterioles, leading to pulmonary hypertension, right heart strain, and death. We administer supplemental oxygen. The lung can continue to develop and heal from injury through early childhood.

Excessive oxygen in the airways leads to Retinopathy of Prematurity and blindness. A relatively straightforward balance can be achieved. The influence of the lung on the retina does not become noticeable until after the damage has started. Damage of a few retina cells impairs growth into more retina cells; a small insult inflicts exponential harm.

Mechanical ventilation increases exposure to bacteria and subsequent pneumonia, increasing the likelihood of death. Prudence guides us to wean the ventilator.

Excessive work by the diaphragm steals energy necessary for muscle growth and brain development. There is now a mismatch in timelines as pneumonia will likely occur before muscle wasting is noted and long before brain development becomes measurable.

The infant's diaphragm contains fast-twitch fibers with fewer fatigue-resistant fibers. A higher breathing rate is adaptive for the infant's chest with its relatively fixed tidal volume. The need for increased blood supply steals blood from other organs, including the brain.

Decreased chest expansion is an interoceptive signal carried by the dorsal vagus nerve to the insular cortex. Decreased tidal volume can cause hypoactive delirium, identified by decreased interactions.

The Neonatologist is aware of this, but the balance is an inductive process of contingent interactions with physiological processes, including different time horizons. Outside physicians are unlikely to grasp these diverse balances.

\section{"The Neonatologist is aware of this, but the balance is an inductive process of contingent interactions with physiological processes, including different time horizons."}

The limits of logic

Abstractions, rules, and protocols do not reliably have the truth, which interferes with their use as truth premises for logical inference. It seems counterintuitive that abstractions limit reason, but even minimal stress impairs cognition $(3,16)$, and abstractions can unintentionally be substituted as concrete information.

Alfred North Whitehead's "Fallacy of Misplaced Concreteness" (54) 52, 57.

In recognition of the dangers of plausible or inductive reasoning, George Pólya (53) characterized the moral qualities of a scientist:

1. The intellectual courage to be ready to revise beliefs

2. The intellectual honesty to change belief for a compelling reason

3. The wise restraint to not change belief wantonly

Doxastic logic and deontic logic are modal logics for belief revision and duty, respectively. 
For Karl Popper, one of the most influential science philosophers of the last century, it was not logical that any positive outcomes from experimental testing could confirm a scientific theory. More problematic was demarcating scientific theory from non-science when some meaningful scientific statements could not be conclusively verified (55). Rather than verifiability, falsifiability became the criterion of whether a theory was testable ( $p$ 95-96) and could then demarcate science from non-science ( $p$ 17-19). The forms must be logically possible to verify and falsify. Popper ( $p 66$ ) differentiated falsifiability, a criterion for the empirical character, from falsification, which requires special rules to regard a system as falsified.

\section{“Popper used 'inconsistency' from classical logic for falsifiability to demarcate empirical science from non-science. To accept inconsistency, equivalent to self- contradiction, is to collapse science."}

Popper (55) ( $p$ 65-66, 71-73) used the two classes of inconsistency and non-contradiction to determine if a theory is 'empirical' or 'falsifiable.' Inconsistency and non-contradiction derive from the classical logic law of non-contradiction, as described above in "Classical Logic." "The importance of the requirement of consistency will be appreciated if one realizes that a self-contradictory system [inconsistent] is uninformative... Any conclusion we please can be derived from it" ( $p$ 73). Popper used 'inconsistency' from classical logic for falsifiability to demarcate empirical science from non-science. To accept inconsistency, equivalent to self-contradiction, is to collapse science.

We continue to experience the influence of Popper when someone offers a single counterexample as proof you are wrong.

"For it can easily be shown that if one were to accept contradictions, then one would have to give up any kind of scientific activity: it would mean a complete break-down of science. This can be shown by proving that if two contradictory sentences are admitted, any sentence whatsoever must be admitted" (56). [The] "law of contradiction; that is to say, upon the demand that contradictions, whenever we discover them, must be eliminated. Critical error-elimination on the scientific level proceeds by way of a conscious search for contradictions" (57).

\section{Karl R. Popper}

Though first-order logic seems like a pillar of science, a presentation of its emergence (58) reveals the limitations of first-order logic and describes the benefits of other logic. John Dewey (59), American philosopher, educator, and psychologist, opposed reliance on first-order logic in the natural sciences, disagreeing with the "assumption that logical and formal principles have a direct material and ontological application, rather than conclusions from empirical evidence."

We can, however, use logical inconsistencies to drive investigation without rejecting available information (60).

\section{Classical First-Order Logic}

Logic is found in philosophy, mathematics, and computer science, which use logic for different purposes, although similar in structure (61). Classical, or classical first-order logic, has entered medical care through the sciences and humanities. Classical first-order logic tells us what follows from a proposition but does not tell us what to believe when to act, how to manage ambiguity or describe our duty to subordinates, family, or the infant. Classical logic can interfere when the Neonatologist uses inductive processes to guide the neonate's care (59).

The scientific method uses classical logic closely related to the study of correct reasoning, making this the presumptive correct logic for science (61). Nevertheless, our experience belies classical logic as correct reasoning, as noted in the vignettes above. Classical logic can impair the extension of a discipline.

At the beginning of the author's (DvS) experience with longterm ventilation, he followed the goal of weaning the child from the ventilator following blood gas evaluation for $\mathrm{O}_{2}$ and $\mathrm{CO}_{2}$. One day a child's grandmother beseeched an LVN to ask if the doctor could leave her grandson on the ventilator. She liked that he smiled, and he had never smiled before. The difference in ventilator management by blood gas versus smile created logical inconsistency in management with staff from various PICUs. Use of the child's affective response appeared to contradict scientific rationality and logic, along with standard respiratory care references that mechanical ventilation was difficult for a patient to tolerate.

Logical pluralism reflects the possibility that other logic can offer solutions (62). Nonclassical logical operators, given the same premises but with different interpretations of 'valid,' can lead to different logical consequences. What one system captures as valid differs from what the other system captures.

"Nonclassical logical operators, given the same premises but with different interpretations of 'valid,' can lead to different logical consequences. What one system captures as valid differs from what the other system captures."

The classical predicate or first-order logic is a formal language expressing propositions in terms of predicates, variables, and quantifiers. Predicates express propositions as statements involving the arguments with the simplest predicates expressing properties of things. A statement makes an assertion that may be true or false contingent on the values of the variables.

An agitated patient with mechanical ventilation can disconnect from the ventilator. Cardiac arrest can come quickly. Rapid administration of sedative medication brings quick resolution. Sedation is not available in the subacute nursing facility. The author taught the subacute RCPs to hand ventilate for patient calm (63). Over time the property of mechanical ventilation shifted from enabling life to enhancing life as children began smiling, talking, and interacting with parents.

\section{Readers can also follow} NEONATOLOGY TODAY via our Twitter Feed @NEOTODAY 
Further extension occurred for infants with weak abdominal musculature who developed acute respiratory distress during a bowel movement. The RCPs learned that mild backpressure, a PEEP of $8 \mathrm{~cm} \mathrm{H}_{2} \mathrm{O}$, would prevent ventilator-related agitation. More significant was the experience of hand ventilation for agitation in children with neurological deficits. After a few breaths, they awaken, older children may begin talking. Discussion with a neuropsychiatrist revealed these children were likely in hypoactive delirium secondary to hypoventilation.

Like the vignette in labor and delivery, the rigidity of classical logic can artificially create tension, corrupt communication, and impair the extension of medical care for the benefit of patients.

\section{Data, statistics, and probability}

A premature infant is a state of nonlinear interactions within each developing organ, between developing organs, and within the environment. Collected and analyzed data creates a body of knowledge for medical evaluation and management. However, numerous nonlinear interactions generate a flux of contradictory information and conflicting data making necessary information inconsistent. Reliance on statistics and probability (for example, evidence-based medicine) is not wrong. We must, though, acknowledge the insight shared by Bob Bea (64) (personal communication):

"The key problem in statistics is the validity of the data that has been gathered."

"The key problem in probability is the potential for changes in conditions in the future that invalidate the data that has been gathered during the past."

"The 'tails of distributions' generally are controlled by 'rare events' = 'outliers.' If the concerns for 'reliability' are primarily concerned with the rare events (for example, commercial aviation and nuclear power generation), then the focus will be on the distribution tails. If the concerns are primarily concerned with frequently occurring events, then the central portions of the distributions are of most interest."

Thus, the problem for the Neonatologist: application of statistics and probability to contradictory and inconsistent information as a particular infant moves from one state of nonlinear interactions to another. Using classical logic to deduce conclusions by inference, reasoning rather than observation lessens our ability to make desirable worlds actual. We lose grasp of the situation.

\section{"Using classical logic to deduce conclusions by inference, reasoning rather than observation lessens our ability to make desirable worlds actual. We lose grasp of the situation."}

\section{Conclusion}

How do we use the information? Information preserves truth and extends the experience. The reasoning for both has not been identified. Those who have accomplished this find it difficult to describe to the scientific rationalist and classical logician. Such experts may suffer heavy criticism.

Fire changed human diet, extended human geography, and changed culture. If HRO describes the ability to control and use dangerous energy for the common good, then early humans developed the first HROs around the use of fire. For bringing fire to humans, Prometheus was punished by the gods.

How do we use the information? The author (DvS) gave a lecture on traffic safety for a state office. The audience came from public safety and healthcare. The author elected to teach 'how to think' using his public safety experience, combat decision methods, and the Affective Domain of Learning, an approach that had engendered criticism from healthcare and EMS administrators. Two senior police detectives approached after the lecture to say it was the first lecture describing and explaining how they think. The title of the lecture? "Where is Prometheus?"

\section{References:}

1. Zundel M, Kokkalis $P$. Theorizing as Engaged Practice. Organization Studies. 2010;31(9-10):1209-27. doi: 10.1177/0170840610374405.

2. van Stralen D. Pragmatic High-Reliability Organization (HRO) During Pandemic COVID-19. Neonatology Today. 2020;15(4):3-9.

3. van Stralen D, Mercer TA. Pragmatic High-Reliability Organizations (HRO) Modulate the Functions of Stress and Fear Behaviors During Pandemic COVID-19: The Stress-FearThreat Cascade. Neonatology Today. 2020;15(10):126-34. doi: 10.51362/neonatology.today/2020101510126134.

4. van Stralen D, Mercer T. High Reliability Organizing (HRO) is the Extension of Neonatology during Pandemic COVID-19. Neonatology Today. 2021;16(5):97-109. doi: 10.51362/neonatology.today/2021516597109.

5. Newton-Smith WH. The rationality of science. New York, NY: Routledge; 2002.

6. Sandberg J, Tsoukas H. Grasping the logic of practice: Theorizing through practical rationality. Academy of management review. 2011;36(2):338-60.

7. Sen A. Rationality and freedom. Cambridge, MA: Harvard University Press; 2002.

8. Weick KE. Organizing for transient reliability: The production of dynamic non-events. Journal of contingencies and crisis

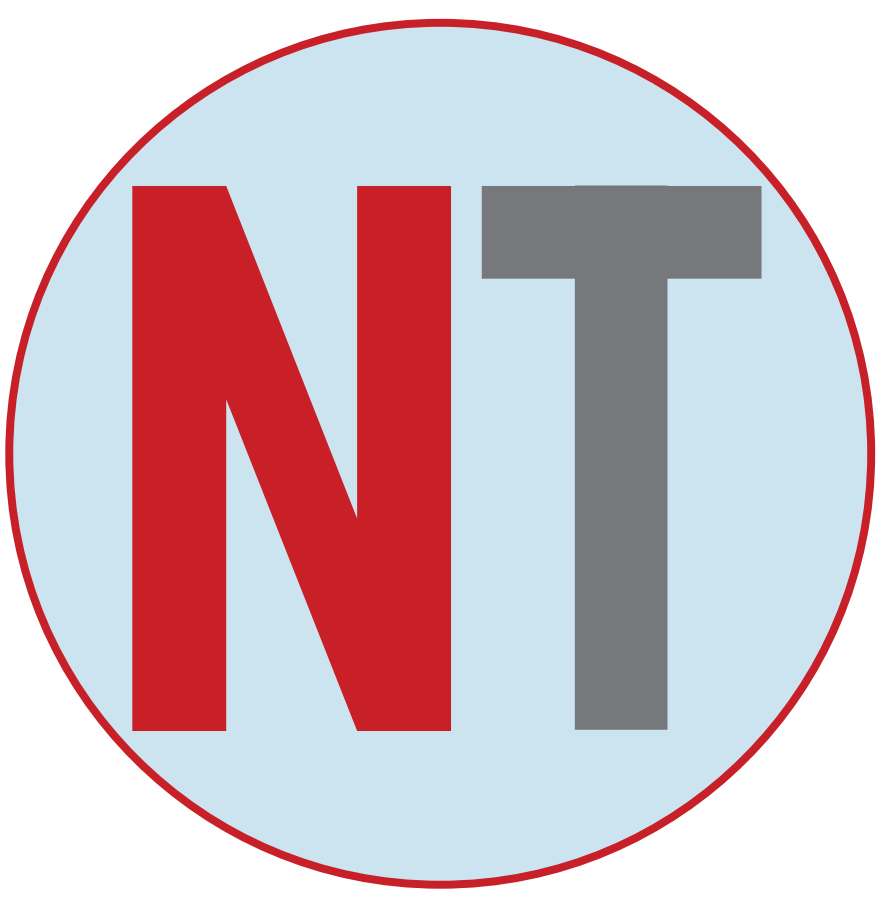


management. 2011;19(1):21-7.

9. van Stralen D. Ambiguity. Journal of Contingencies and Crisis Management. 2015;23(2):47-53. doi: 10.1111/14685973.12082.

10. van Stralen D, Gambino W. Error as a Faulty Failure Signal. Neonatology Today. 2020;15(9):114-7. doi: 10.51362/neonatology.today/20209159114117.

11. van Stralen D, Mercer TA. Pandemic COVID-19, the HighReliability Organization (HRO), and the Ecology of Fear. Neonatology Today. 2020;15(12):129-38. doi: 10.51362/ neonatology.today/2020121512129138.

12. Pauly D. Anecdotes and the shifting baseline syndrome of fisheries. Trends in Ecology \& Evolution. 1995;10(10):430.

13. van Stralen D, Mercer TA. The Nature of Neonatal Experience during Pandemic COVID-19. Neonatology Today. 2021;16(3):87-97. doi: 10.51362/neonatology.today/202131638797.

14. Mason JW. A review of psychoendocrine research on the pituitary-adrenal cortical system. Psychosomatic medicine. 1968;30(5):576-607.

15. Gagnon SA, Wagner AD. Acute stress and episodic memory retrieval: neurobiological mechanisms and behavioral consequences. Annals of the New York Academy of Sciences. 2016;1369(1):55-75.

16. Arnsten AF. Stress signalling pathways that impair prefrontal cortex structure and function. Nat Rev Neurosci. 2009;10(6):410-22. Epub 2009/05/21. doi: 10.1038/nrn2648. PubMed PMID: 19455173; PubMed Central PMCID: PMCPMC2907136.

17. Shields GS, Sazma MA, Yonelinas AP. The effects of acute stress on core executive functions: $A$ meta-analysis and comparison with cortisol. Neuroscience \& Biobehavioral Reviews. 2016;68:651-68.

18. Weick KE. The collapse of sensemaking in organizations: The Mann Gulch disaster. Administrative science quarterly. 1993;38(4):628-52.

19. Aristotle. Aristotle's Nicomachean ethics. Chicago, IL: University of Chicago Press; 2011.

20. van Stralen D, Mercer TA. High-Reliability Organizing (HRO) in the COVID-19 Liminal Zone: Characteristics of Workers and Local Leaders. Neonatology Today. 2021;16(4):90-101. doi: 10.51362/neonatology.today/2021416490101.

21. Magee RR. Strategic leadership primer. Army War Coll Carlisle Barracks PA, 1998.

22. Arnold III AV. Strategic visioning: What it is and how it's done. ARMY WAR COLL CARLISLE BARRACKS PA, 1991.

23. van Stralen $D$, Inozu B, Byrum S. High Reliability for a Highly Unreliable World: Preparing for Code Blue through Daily Operations in Healthcare. North Charleston, SC: CreatSpace Publishing; 2017.

24. van Stralen D, McKay S, Williams GT, Mercer TA. Tactical Improvisation: After-Action/ Comprehensive Analysis of the Active Shooter Incident Response by the San Bernardino City Fire Department December 2, 2015. San Bernardino, CA: San Bernardino County Fire Protection District; 2018.

25. McKay S, James J, Greg S, Dominick B, Bryan H, Ditzel R, et al. Refining Operational Vertical Mobility. Journal of High Threat \& Austere Medicine. 2021:19. doi: 10.33553/jhtam. v3i1.33.

26. Szakolczai A. Liminality and experience: Structuring transitory situations and transformative events. International Po- litical Anthropology. 2009;2(1):141-72.

27. Weick KE. Organizing for Transient Reliability: The Production of Dynamic Non-Events. Journal of Contingencies and Crisis Management. 2011;19(1):21-7. doi: 10.1111/j.14685973.2010.00627.x.

28. Fristrup KM. A history of character concepts in evolutionary biology. In: Wagner GP, editor. The character concept in evolutionary biology. San Diego, CA: Academic Press; 2001. $p$. 13-36.

29. Sih A, Bell AM, Johnson JC, Ziemba RE. Behavioral syndromes: an integrative overview. The quarterly review of biology. 2004;79(3):241-77.

30. Braica AP. Particularities of the organizational culture in the higher education establishments in Romania. Scientific Papers Series Management, Economic Engineering in Agriculture and Rural Development. 2014;14(2):35-40.

31. Caspi A, Bem DJ, Elder Jr GH. Continuities and consequences of interactional styles across the life course. Journal of personality. 1989;57(2):375-406.

32. Heggie V. Experimental physiology, Everest and oxygen: from the ghastly kitchens to the gasping lung. The British Journal for the History of Science. 2012;46(1):123-47. doi: 10.1017/s0007087412000775.

33. Weick KE. Enacted sensemaking in crisis situations Journal of management studies. 1988;25(4):305-17.

34. Bobko JP, Sinha M, Chen D, Patterson S, Baldridge T, Eby $M$, et al. A Tactical Medicine After-action Report of the San Bernardino Terrorist Incident. West J Emerg Med. 2018;19(2):287-93. Epub 2018/03/22. doi: 10.5811/ westjem.2017.10.31374. PubMed PMID: 29560056; PubMed Central PMCID: PMCPMC5851501 are required to disclose all affiliations, funding sources and financial or management relationships that could be perceived as potential sources of bias. No author has professional or financial relationships with any companies that are relevant to this study. There are no conflicts of interest or sources of funding to declare.

35. van Stralen $D$, McKay $S D$, Inaba $K$, Hartwig $M$, Mercer TA. Comment on "A Tactical Medicine After-action Report of the San Bernardino Terrorist Incident". West J Emerg Med. 2018;19(5):825-6. Epub 2018/09/12. doi: 10.5811/ westjem.2018.6.39216. PubMed PMID: 30202494; PubMed Central PMCID: PMCPMC6123096 are required to disclose all affiliations, funding sources and financial or management relationships that could be perceived as potential sources of bias. No author has professional or financial relationships with any companies that are relevant to this study. There are no conflicts of interest or sources of funding to declare.

36. Rocha V, Winton R, Esquivel P, Nelson LJ. Boy, 8, and teacher slain in San Bernardino school shooting; gunman kills himself. Los Angeles Times. 2017 April 10, 2017;Sect. California.

37. Weick KE. Enactment and Organizing. The Social Psychology of Organizing. Second ed. New York, NY: McGraw-Hill, Inc.; 1979. p. 147-69.

38. van Stralen D, Mercer TA. High Altitude Climbing, High Reliability, COVID-19, and the Power of Observation. Neonatology Today. 2021;16(1):68-79. doi: 10.51362/neonatology. today/20211616879.

39. Elmes $M$, Frame $B$. Into hot air: $A$ critical perspective on Everest. Human Relations. 2008;61(2):213-41. doi: 
$10.1177 / 0018726707087785$.

40. Dreyfus SE, Dreyfus HL. The Scope, Limits, and Training Implications of Three Models of Aircraft Pilot Emergency Response Behavior. Berkeley, CA: University of California, Berkeley, Center OR; 1979 February 1979. Report No.: ORC 79-2 Contract No.: ORC 79-2.

41. Dreyfus HL, Dreyfus SE. The Psychic Boom: Flying Beyond the Thought Barrier. Berkeley, CA: University of California, Berkeley, Center OR; 1979 March 1979. Report No.: ORC 79-2.

42. Benner P. From novice to expert. American Journal of nursing. 1982;82(3):402-7.

43. Benner P. Using the Dreyfus model of skill acquisition to describe and interpret skill acquisition and clinical judgment in nursing practice and education. Bulletin of science, technology \& society. 2004;24(3):188-99.

44. Online Etymology Dictionary.

45. Raatikainen P. Gödel's Incompleteness Theorems. In: Zalta EN, editor. The Stanford Encyclopedia of Philosophy: Metaphysics Research Lab, Stanford University; 2021.

46. Whitehead AN, Russell B. Principia Mathematica. 2nd ed. London, UK: Cambrdge University Press; 1927.

47. McGill VJ. Concerning the laws of contradiction and excluded middle. Philosophy of Science. 1939;6(2):196-211.

48. Loparić A, da Costa NC. Paraconsistency, paracompleteness, and valuations. Logique et analyse. 1984;27(106):11931.

49. Ciuciura J. Paraconsistency and Paracompleteness. Logical Investigations. 2019;25(2):46-60. doi: 10.21146/2074-14722019-25-2-46-60.

50. Ciucci $D$, Dubois $D$. Three-valued logics, uncertainty management and rough sets. In: Peters JF, Skowron A, editors. Transactions on Rough Sets XVII. Lecture Notes in Computer Science. Berlin, Germany: Springer-Verlag; 2014. p. 1-32.

51. da Costa NC, Krause D, Bueno O. Paraconsistent logics and paraconsistency. In: Jacquette $D$, editor. Philosophy of logic. Handbook of the Philosophy of Science. Amsterdam, The Netherlands: Elsevier; 2007. p. 791-911.

52. Euler L. Opera Omnia, ser. 1, vol. 2. In: Pólya G, editor. Mathematics and plausible reasoning: Induction and analogy in mathematics. 1. Princeton, NJ: Princeton University Press; 1761.

53. Pólya G. Mathematics and plausible reasoning: Induction and analogy in mathematics. Princeton, NJ: Princeton University Press; 1954.

54. Whitehead AN. Science and the modern world. Cambridge, UK: Cambridge University Press; 1926.

55. Popper KR. The logic of scientific discovery. English ed. New York, NY: Routledge; 1959.

56. Popper KR. What is Dialectic? . Mind. 1940;49(196):403-26.

57. Popper KR. Objective knowledge: Oxford University Press Oxford; 1972.

58. Ewald W. The Emergence of First-Order Logic. In: Zalta EN, editor. The Stanford Encyclopedia of Philosophy: Metaphysics Research Lab, Stanford University; 2019.

59. Dewey J. The sphere of application of the excluded middle. The Journal of Philosophy. 1929;26(26):701-5.

60. Bueno O. Why inconsistency is not hell: Making room for inconsistency in science. In: Olsson EJ, editor. Knowledge and inquiry: Essays on the Pragmatism of Isaac Levi. New
York, NY: Cambridge University Press; 2006. p. 70-86.

61. Shapiro S, Kissel TK. Classical Logic. In: Zalta EN, editor. The Stanford Encyclopedia of Philosophy. Spring 2021 Edition ed: Metaphysics Research Lab, Stanford University; 2021.

62. Russell G. Logical Pluralism. In: Zalta EN, editor. The Stanford Encyclopedia of Philosophy: Metaphysics Research Lab, Stanford University; 2021.

63. van Stralen D, Westmoreland T. Management of a Mechanical Ventilator Using Patient Calm as an Endpoint. Special Operations Medical Association Scientific Assembly (SOMSA); December 8-11, 2014; Tampa, Florida: Special Operations Medical Association (SOMA); 2014.

64. Prud'homme A. Bob Bea, the Master of Disaster. Men's Journal 2013:72-5.

Disclosures: The authors have no relevant disclosures

NT
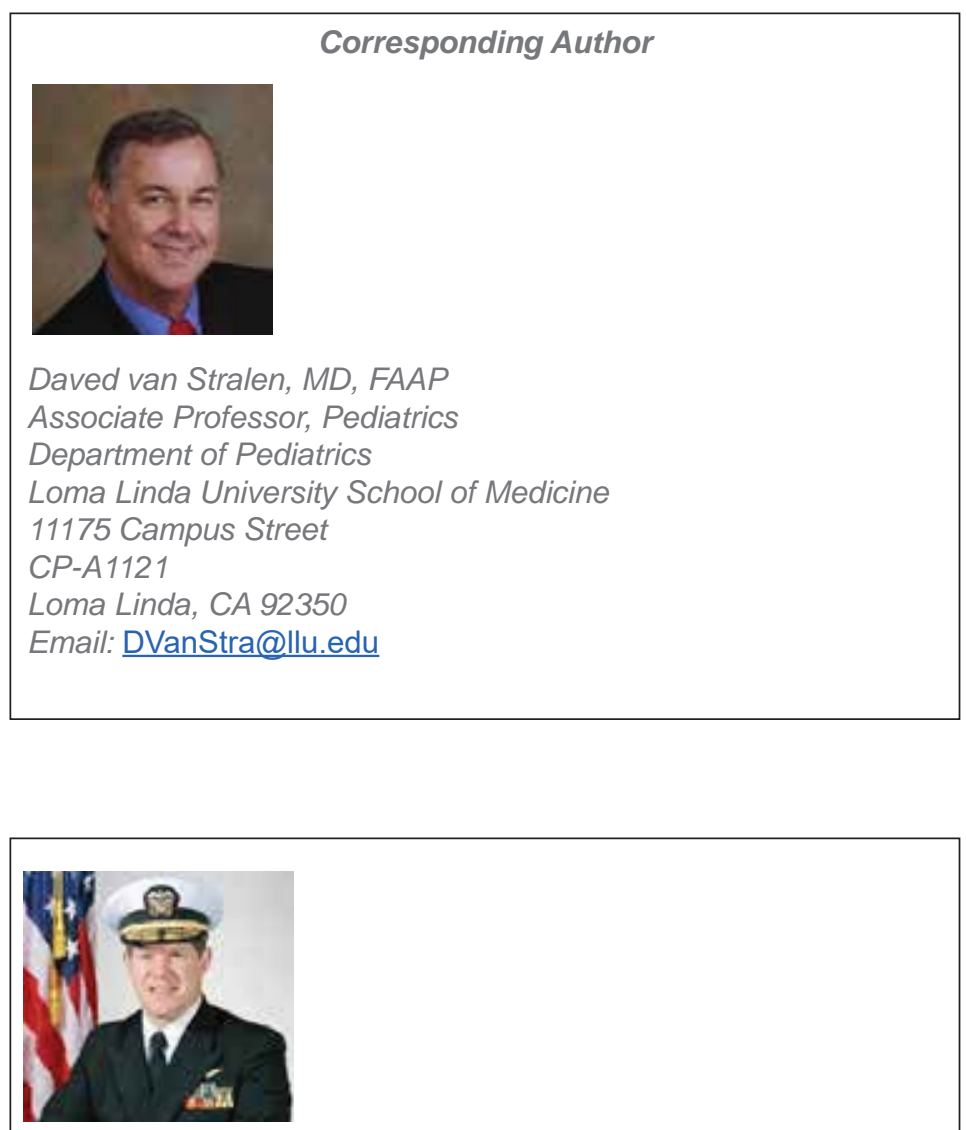

Thomas A. Mercer

Rear Admiral

United States Navy (Retired) 


\section{Acknowledgments}

Karl Weick, Rensis Likert Distinguished University Professor of Organizational Behavior and Psychology, Emeritus, University of Michigan

Sean McKay, Element Rescue, LLC

Errol van Stralen, Ancora Education

Steve Papenfuhs, Insight Training Strategies

Tim Watson, Mission Pediatrics

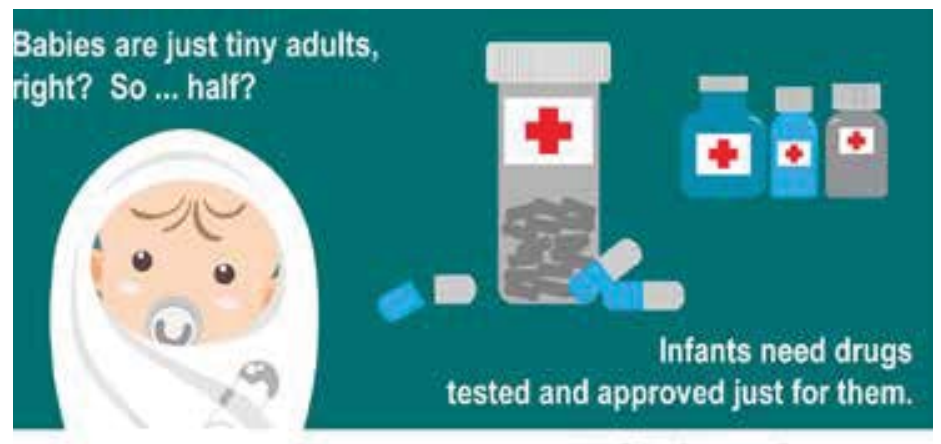

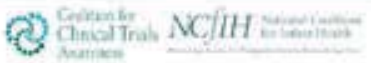

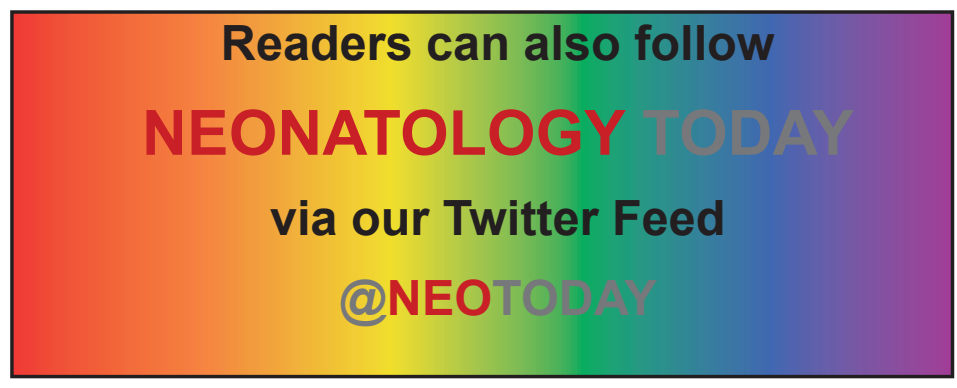

\section{New subscribers are always welcome!} NEONATOLOGY TODAY

To sign up for a free monthly subscription, just click on this box to go directly to our subscription page
OPIOIDS and NAS

When reporting on mothers, babies, and substance use

\section{LANGUAGE MATTERS}

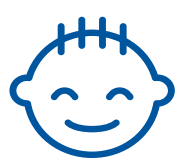

I am not an addict.

I was exposed to substances in utero. I am not addicted. Addiction is a set of behaviors associated with having a Substance Use Disorder (SUD).

\section{I was exposed to opioids.}

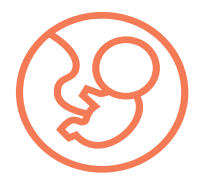

While I was in the womb my mother and I shared a blood supply. I was exposed to the medications and substances she used. I may have become physiologically dependent on some of those substances.

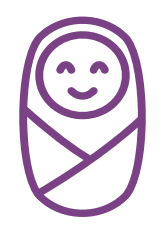

NAS is a temporary and treatable condition.

There are evidence-based pharmacological and non-pharmacological treatments for Neonatal Abstinence Syndrome.

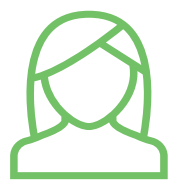

\section{My mother may have a SUD.}

She might be receiving Medication-Assisted Treatment (MAT). My NAS may be a side effect of her appropriate medical care. It is not evidence of abuse or mistreatment.

My potential is limitless.

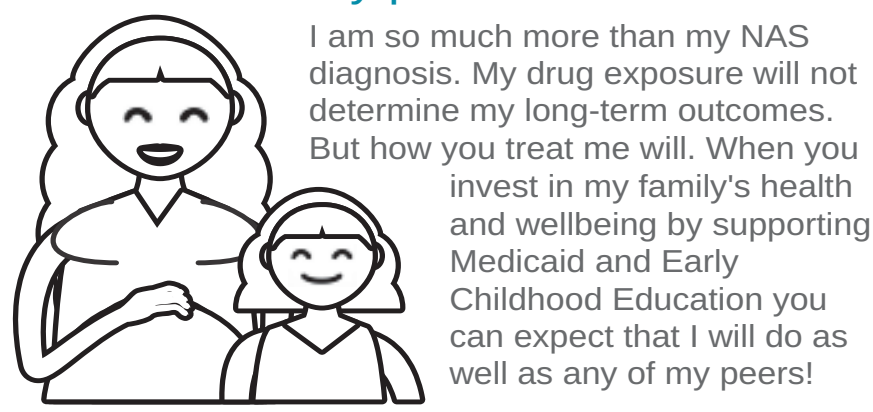

Learn more about

Neonatal Abstinence Syndrome at wWW.nationalperinatal.org 